\title{
PREVALÊNCIA E FATORES ASSOCIADOS ÀS ANOMALIAS CONGÊNITAS EM RECÉM-NASCIDOS
}

\section{Prevalence and associated factors of congenital abnormalities in newborns \\ Prevalencia y factores asociados con las anomalías congénitas de recién-nacidos}

Elzo Pereira Pinto Junior

Universidade Federal da Bahia - ISC - UFB - Salvador (BA) - Brasil

Leandro Alves da Luz

Universidade Federal da Bahia - ISC - UFB - Salvador (BA) - Brasil

Marina Aguiar Pires Guimarães

Universidade Federal de Minas Gerais - UFMG - Belo Horizonte (MG) - Brasil

Lívia Teixeira Tavares

Hospital Estadual da Criança - HEC - Feira de Santana (BA) - Brasil

Tatiana Ribeiro Santos Brito

Maternidade de Referência Prof. José Maria de Magalhães Neto - Salvador (BA) - Brasil

\section{Gabriela Di Filippo Souza}

Universidade Federal da Bahia - ISC - UFB - Salvador (BA) - Brasil

\section{RESUMO}

Objetivo: Descrever a prevalência e analisar os fatores associados às anomalias congênitas em recém-nascidos (RN). Métodos: Estudo transversal com base em 33.141 declarações de nascidos vivos (DNVs) de bebês cujas mães eram residentes em Salvador, Bahia, em 2014. Inicialmente foi realizada uma análise descritiva, seguida de análise bivariada da associação entre anomalias congênitas e características maternas, gestacionais e dos RN, com estimação de razão de prevalência (RP) e intervalos de confiança de 95\% (IC95\%). Após a análise bivariada, procedeu-se a análise de regressão logística multivariada. Resultados: A prevalência de anomalias congênitas foi 1,0\%, sendo as principais causas condições ligadas ao sistema osteomioarticular (52,1\%). A análise multivariada evidenciou maior prevalência de anomalias em recém-nascidos do sexo masculino ( $\mathrm{RP}=1,40$; IC95\%:1,12-1,74), com baixo peso ao nascer ( $\mathrm{RP}=2,34$; IC95\%:1,77 - 3,08) e escore de Apgar insatisfatório ao quinto minuto ( $\mathrm{RP}=3,34$; IC95\%: 2,30 - 4,82), cujas mães tinham menos de 18 anos ou mais de 35 anos ( $\mathrm{RP}=1,69$; IC95\%: 1,17-2,43) e realizaram parto cesárea ( $\mathrm{RP}=1,46$; IC95\%:1,17-1,83). Conclusão: Encontrou-se baixa prevalência de malformações congênitas, sendo esse desfecho associado às mães em extremo de idade e quadro clínico adverso ao nascimento, como baixo peso e Apgar insatisfatório.

Descritores: Anomalias Congênitas; Fatores de Risco; Recém-nascido; Epidemiologia; Sistemas de Informação em Saúde.

\section{ABSTRACT}

Objective: To describe the prevalence and analyze the associated factors of congenital abnormalities in newborns (NBs). Methods: Crosssectional study based on 33,141 Live Birth Certificates (LBCS) of babies whose mothers were living in Salvador, Bahia, in 2014. A descriptive analysis was initially performed, followed by bivariate analysis of the association between congenital abnormalities and maternal, pregnancy, and newborns characteristics, with estimation of prevalence ratio (PR) and 95\% confidence intervals (95\% CI). After the bivariate analysis, the multivariate logistic regression was performed. Results: The prevalence of congenital abnormalities was $1.0 \%$, and the leading causes were conditions related to the musculoskeletal system (52.1\%). The multivariate analysis evidenced higher prevalence of abnormalities in male newborns ( $P R=1.40$; 95\%CI: 1.12-1.74), with low birth weight ( $P R=2.34$; 95\% CI: 1.77-3.08) and unsatisfactory five-minute Apgar score (PR=3.34; 95\%CI: 2.30-4.82), whose mothers were under 18 or over 35 years of age ( $P R=1.69 ; 95 \% C I: 1.17-2.43)$ and underwent cesarean delivery ( $P R=1.46$; 95\%CI: 1.17-1.83). Conclusion: A low prevalence of congenital abnormalities was found, and this outcome was associated with mothers at an extreme age and adverse clinical condition at birth, such as low weight and unsatisfactory Apgar score.

Descriptors: Congenital Abnormalities; Risk Factors; Newborn; Epidemiology; Health Information Systems. 


\section{RESUMEN}

Objetivo: Describir la prevalencia y analizar los factores asociados con las anomalías congénitas de recién-nacidos (RN). Métodos: Estudio transversal basado en 33.141 declaraciones de nacidos vivos (DNVs) de bebés cuyas las madres vivian en Salvador de Bahia en 2014. A principio fue realizado un análisis descriptivo seguido de un análisis bivariado de la asociación entre las anomalías congénitas y las características de las madres, de las gestaciones y de los $R N$ con la estimación de una razón de prevalencia (RP) e intervalos de confianza del 95\% (IC95\%). Después del análisis bivariado se dió el análisis de regresión logística multivariada. Resultados: La prevalencia de anomalías congénitas fue del 1,0\%, teniendo como las principales causas las condiciones asociadas al sistema osteomioarticular (52,1\%). El análisis multivariado evidenció mayor prevalencia de anomalias en recién-nacidos del sexo masculino (RP=1,40; IC95\%:1,12-1,74), con bajo peso al nacer ( $R P=2,34$; IC95\%:1,77 - 3,08) y la puntuación del Apgar insatisfactoria en el quinto minuto $(R P=3,34 ;$ IC95\%: 2,30 - 4,82), cuyas madres tenían menos de 18 años o más de 35 años ( $R P=1,69$; IC95\%: 1,17-2,43) y que habian realizado el parto por cesárea (RP=1,46; IC95\%:1,17-1,83). Conclusión: Se encontró baja prevalencia de mal formaciones congénitas que estuvo asociado a las madres con extrema edad y cuadro clínico adverso al nacimiento, bajo peso y el Apgar insatisfactorio.

Descriptores: Anomalias Congénitas; Factores de Riesgo; Recién Nacido; Epidemiología; Sistemas de Información en Salud.

\section{INTRODUÇÃO}

As anomalias congênitas ou malformações são desordens estruturais ou funcionais que ocorrem no período do desenvolvimento fetal e podem ser detectadas durante o pré-natal, parto ou após o nascimento ${ }^{(1,2)}$. No mundo, estima-se que $3 \%$ das crianças são afetadas por malformações congênitas ${ }^{(3)}$. Em 2013, das 2,76 milhões de mortes registradas no período neonatal, aproximadamente $10 \%$ foram por malformações congênitas ${ }^{(2)}$. No Brasil, as anomalias congênitas ocupam o segundo lugar na lista das causas de óbitos infantis, atingindo cerca de 3\% do total de nascidos vivos e, a cada ano, são registrados 60.000 casos dessas doenças ${ }^{(4)}$.

Estimativas apontam que $60 \%$ dos transtornos congênitos possuem etiologia desconhecida. Entretanto, estudos têm demonstrado associação entre defeitos congênitos e fatores ambientais, genéticos e condições maternas, como o uso de determinadas drogas ilícitas e tabagismo. Outros fatores, como assistência materno-infantil inadequada e baixo nível socioeconômico, também são apontados como fatores associados às malformações congênitas, principalmente nos países pobres e em desenvolvimento ${ }^{(5-8)}$. Pesquisas recentes destacam a preocupação com o aumento da incidência de microcefalia relacionada à epidemia do vírus Zika (ZIKV) no Brasil ${ }^{(9)}$, haja vista que puérperas sem exposição prévia aos fatores de risco já conhecidos, mas infectadas por esse vírus durante o primeiro trimestre da gravidez, tiveram bebês diagnosticados com microcefalia ${ }^{(10)}$. Nesse contexto, recente revisão sistemática evidenciou aumento no número de casos de recém-nascidos com malformações congênitas, especialmente microcefalia, devido a infecções por Zika em gestantes residentes na Região Nordeste do Brasil(11).

Além da elevada mortalidade, as anomalias congênitas são causas frequentes de incapacidades físicas nos recém-nascidos, muitas das quais são irreversíveis e provocam sofrimento psicológico aos seus pais ou cuidadores. As repercussões sociais das malformações congênitas exigem a organização de uma rede de cuidados continuados por parte dos serviços de saúde, de modo a garantir assistência integral, que deve ser ofertada a partir de equipes multiprofissionais que forneçam reabilitação física aos bebês e o apoio psicológico ao núcleo familiar ${ }^{(4,8)}$.

No Brasil, os registros de nascimento e outros desfechos de interesse para o estudo de alguns problemas de saúde da população são armazenados em Sistemas de Informação em Saúde, geridos pelo Departamento de Informática do Sistema Único de Saúde (DATASUS). Um desses sistemas é o Sistema de Informações sobre Nascidos Vivos (SINASC), criado em 1990, com o objetivo de reunir informações coletadas sobre as condições de nascimento dos recém-nascidos (Escore de Apgar, peso, presença de anomalias congênitas), além de dados referentes à mãe, ao pré-natal e ao parto. Esse sistema é alimentado pela Declaração de Nascido Vivo (DNV), de preenchimento obrigatório em todo o território nacional ${ }^{(12)}$. Além da sua função administrativa, o SINASC também fornece dados que permitem a pesquisa e o monitoramento de características de saúde da população materno-infantil. A partir de seus dados, é possível subsidiar as intervenções relacionadas à saúde da mulher e da criança no âmbito do Sistema Único de Saúde (SUS). No Brasil, as bases de dados secundários têm sido cada vez mais utilizadas em pesquisas e esse interesse cresce à medida que a disponibilidade e qualidade dos dados são melhoradas ${ }^{(13)}$.

No contexto da atenção à saúde materno-infantil, as ações de promoção à saúde são indispensáveis para a qualidade da assistência desde o pré-natal até o desenvolvimento da criança na primeira infância. Nesse sentido, recém-nascidos com anomalias congênitas e suas famílias precisam ser alvos de um conjunto de atividades que vão além do cuidado clínico e ambulatorial, incorporando outras ações de âmbito intersetorial e com uma abordagem ampliada às necessidades de saúde dessa população.

Conhecer a prevalência e os fatores associados às anomalias congênitas deve ser encarado como uma prioridade no Brasil, haja vista que essas doenças representam uma das principais causas de mortalidade infantil e geram graves repercussões na vida 
da criança e de sua família. Desse modo, o objetivo deste estudo foi descrever a prevalência e analisar os fatores associados à anomalia congênita em recém-nascidos.

\section{MÉTODOS}

Trata-se de um estudo epidemiológico do tipo transversal, exploratório, com dados secundários de acesso público. As fontes dos dados foram as declarações de nascidos vivos de mães residentes em Salvador, entre janeiro e dezembro de 2014 , disponibilizados no Sistema de Informação sobre Nascidos Vivos, gerenciado pelo Departamento de Informática do Sistema Único de Saúde. A escolha desse período de tempo deu-se por conta da disponibilidade dos dados no SINASC, haja vista que, no momento da coleta das informações, tratava-se do ano mais recente.

A limpeza do banco de dados foi realizada antes da análise estatística, e teve como objetivo garantir a seleção das DNVs que continham informações válidas para a variável do desfecho e demais covariáveis utilizadas nesta pesquisa. Entre janeiro e dezembro de 2014, foram notificados 36.634 nascimentos em Salvador. Inicialmente, realizou-se a descrição das variáveis para identificar a presença de valores ausentes (missing values) e foram excluídas as observações que não continham informação sobre a presença de anomalias congênitas $(n=1503)$. Na sequência, foram excluídas as declarações que não continham dados sobre tipo de parto, consultas pré-natais, sexo do recém-nascido, índice de Apgar ao quinto minuto, tipo de gestação e tipo de gravidez. Ao final da rotina de limpeza, restaram para a análise 33.141 DNVs, que representavam 90,4\% de todos os nascimentos disponíveis no SINASC. Ressalta-se que foram feitas análises descritivas e de associação de interesse antes e após as exclusões, não sendo notadas diferenças nos percentuais nem nas medidas de associação. Esse procedimento indica que a limpeza das variáveis não resultou em viés de seleção, ao passo que garantiu o mesmo número de observações para todas as variáveis analisadas.

As variáveis selecionadas para análise neste estudo foram dicotomizadas e divididas em dois grupos: aspectos maternos, da gestação e parto, e características do recém-nascido. O grupo de características maternas foi composto por: idade da mãe ("18 a 35 anos" ou "< 18 anos ou $>35$ anos"), tipo de gravidez ("única" ou "múltipla") e tipo de parto ("vaginal” ou "cesárea"). As variáveis relacionadas aos recém-nascidos foram: sexo do RN ("feminino" ou "masculino"), prematuridade ("sim” ou "não"), baixo peso ao nascer ("sim” ou "não"), escore de Apgar insatisfatório aos 5 minutos ("sim” ou "não") e presença de anomalia congênita ("sim” ou “não"). A presença de anomalia congênita considerou o diagnóstico de pelo menos um dos seguintes códigos da 10 Classificação Internacional de Doenças (CID-10): Espinha bífida (Q05); Outras malformações congênitas do sistema nervoso (Q00-Q04,Q06-Q07); Malformações congênitas do aparelho circulatório (Q20-Q28); Fenda labial e fenda palatina (Q35-Q37); Ausência, atresia e estenose do intestino delgado (Q41); Outras malformações congênitas do aparelho digestivo (Q38-Q40,Q42-Q45); Testículo não-descido (Q53); Outras malformações do aparelho geniturinário (Q50-Q52,Q54-Q64); Deformidades congênitas do quadril (Q65); Deformidades congênitas dos pés (Q66); Outras malformações e deformidades congênitas do aparelho osteomuscular (Q67-Q79); Outras malformações congênitas (Q10-Q18,Q30-Q34,Q80-Q89); Anomalias cromossômicas não classificadas em outra parte (Q90-Q99); Hemangioma e linfangioma (D18); Anomalias dentofaciais (K07); Outras afecções comprometendo o tegumento específicas do feto e do recém-nascido (P83).

Definiu-se prematuridade como gestação com duração inferior a 37 semanas. Considerou-se baixo peso ao nascer (BPN) todos os bebês que apresentavam peso inferior a 2.500 gramas no nascimento. $\mathrm{O}$ escore de Apgar ao $5^{\circ}$ minuto com valor menor do que sete indicava escore insatisfatório. A "presença de anomalia" congênita foi escolhida como desfecho do estudo (variável dependente). Além dessas variáveis, também foram descritas as principais causas de anomalias nos recém-nascidos.

A análise descritiva dos dados contou com cálculo de frequência absoluta e relativa de todas as variáveis. Estimou-se a prevalência total de anomalia congênita em recém-nascidos e a sua prevalência nos subgrupos de variáveis relacionadas às características maternas e ao recém-nascido. Também foram estimadas as razões de prevalência (RP) e os respectivos intervalos de confiança de 95\% (IC95\%). Além disso, utilizou-se o teste de qui-quadrado de Pearson para testar a associação das covariáveis em relação à presença de anomalia, sendo consideradas estatisticamente significativas aquelas associações cujo valor de $\mathrm{p}<0,05$.

Além da análise bivariada, modelos multivariados foram construídos para descrever os fatores associados à ocorrência de anomalias congênitas em recém-nascidos. Optou-se pela modelagem por meio da regressão logística backward stepwise, sendo utilizadas como critério de inclusão no modelo as variáveis que se associaram com o desfecho com valor de $\mathrm{p}<0,1 \mathrm{e}$ permanência no modelo se valor de $\mathrm{p}<0,05$. Para a escolha do modelo final, que representa o modelo que melhor ajusta os dados do estudo, foram realizados o teste da razão de verossimilhaça e o teste qui-quadrado de Pearson para qualidade do ajuste do modelo. Por se tratar de estudo com desfecho de baixa prevalência, admite-se o uso da odds ratio (OR) como medida numericamente equivalente à razão de prevalência, o que justifica o uso da regressão logística neste estudo, que apresenta desenho transversal.

Tendo em vista que este estudo foi realizado com dados secundários de acesso público e que tais dados não permitem a identificação pessoal das mães ou dos recém-nascidos, não necessitou de aprovação de Comitê de Ética em Pesquisa com seres humanos, estando em concordância com a Resolução 466/12 do Conselho Nacional de Saúde. 


\section{RESULTADOS}

A análise das características maternas, da gravidez e do parto evidenciou que $99,0 \%$ das mães dos nascidos vivos em Salvador tinham entre 18 e 35 anos de idade, 97,6\% apresentaram gravidez única e 53,0\% dos nascimentos ocorreram por parto cesárea (Tabela I).

Tabela I - Características das mães, da gravidez e do tipo de parto, Salvador, Bahia, 2014.

\begin{tabular}{lcc}
\hline Variáveis & $\mathbf{n}$ & $\mathbf{\%}$ \\
\hline Idade da mãe & 32.809 & 99,0 \\
$18-35$ anos & 332 & 1,0 \\
$\quad<18$ anos ou $>35$ anos & & \\
Tipo de gravidez & 32.334 & 97,6 \\
$\quad$ Única & 807 & 2,4 \\
$\quad$ Múltipla & & 47,0 \\
Tipo de parto & 15.587 & 53,0 \\
$\quad$ Vaginal & 17.554 & \\
Cesáreo
\end{tabular}

Fonte: SINASC/Ministério da Saúde.

Em relação às características do recém-nascido, observou-se que 51,0\% deles eram do sexo masculino e 10,4\% nasceram prematuros. O baixo peso ao nascer foi observado em $9,4 \%$ dos bebês e 2,7\% dos RN apresentaram escore de Apgar insatisfatório ao quinto minuto de vida. A prevalência de anomalia congênita foi 1,0\%. As principais causas de malformações nos 332 nascidos vivos com anomalias congênitas se relacionaram às condições ligadas ao sistema osteomioarticular $(52,1 \%)$ e ao aparelho geniturinário $(11,4 \%)$ (Tabela II).

Tabela II - Características e causas de anomalias congênitas em recém-nascidos, Salvador, Bahia, 2014.

\begin{tabular}{lcc}
\hline Variáveis & $\mathbf{n}$ & $\mathbf{\%}$ \\
\hline Sexo do recém-nascido & 16.225 & 49,0 \\
$\quad$ Feminino & 16.916 & 51,0 \\
$\quad$ Masculino & & \\
Prematuridade & 29.708 & 89,6 \\
$\quad$ Não & 3.433 & 10,4 \\
Sim & & \\
Baixo peso ao nascer (<2500g) & 30.043 & 90,6 \\
Não & 3.098 & 9,4 \\
Sim & & \\
Apgar insatisfatório ao 5 min. $^{\text {Não }}$ & 32.241 & 97,3 \\
Sim & 900 & 1,7 \\
Anomalia congênita & & \\
Não & 32.809 & 99,0 \\
Sim & 332 & 1,0 \\
Causas de malformação congênita (n=332) & & \\
Malformações do Sistema Osteomioarticular & 173 & $52,1 \%$ \\
Malformações do Aparelho Geniturinário & 38 & $11,4 \%$ \\
Malformações do Sistema Nervoso & 21 & $6,3 \%$ \\
Malformações do Aparelho Digestivo & 21 & $6,3 \%$ \\
Malformações do Aparelho Circulatório & 16 & $4,9 \%$ \\
Outras malformações congênitas & 63 & $19,0 \%$ \\
\hline Fo: & & \\
\hline
\end{tabular}

Fonte: SINASC/Ministério da Saúde. 
A prevalência de anomalia congênita foi maior em recém-nascidos cujas mães tinham menos de 18 anos ou mais de 35 anos de idade $(\mathrm{RP}=1,78$; IC95\%:1,24-2,54), com gravidez múltipla $(\mathrm{RP}=2,30$; IC95\%:1,44-3,67) e parto cesárea $(\mathrm{RP}=1,47$; IC95\%: 1,18-1,83). Em relação às características dos recém-nascidos, observaram-se maiores prevalências de anomalias em bebês do sexo masculino ( $\mathrm{RP}=1,38$, IC95\%: 1,11 - 1,72), prematuros ( $\mathrm{RP}=2,35, \mathrm{IC} 95 \%$ : 1,81-3,05), que apresentaram baixo peso ao nascer ( $\mathrm{RP}=2,88$, IC95\%: 2,23 - 3,71) e escore de Apgar insatisfatório ao quinto minuto de vida $(\mathrm{RP}=4,63$, IC95\%: 3,32 - 6,45). Destaca-se que, na análise bivariada, todas as características descritas se associaram de modo estatisticamente significante com a presença de anomalias congênitas nos recém-nascidos (Tabela III).

Tabela III - Prevalência e razões de prevalência (RP) de anomalia congênita, segundo as idades materna, aspectos da gravidez e do parto e características dos recém-nascidos de Salvador, Bahia, 2014.

\begin{tabular}{|c|c|c|c|c|c|}
\hline & \multicolumn{2}{|c|}{ Anomalia Congênita } & \multirow[b]{2}{*}{$\mathbf{R P}$} & \multirow[b]{2}{*}{ IC } & \multirow[b]{2}{*}{ valor de $p$} \\
\hline & $\mathbf{n}$ & $\%$ & & & \\
\hline Idade da mãe & & & & & 0,001 \\
\hline 18-35 anos & 299 & 1,0 & 1,00 & - & \\
\hline$<18$ anos ou $>35$ anos & 33 & 1,7 & 1,78 & $(1,24-2,54)$ & \\
\hline Tipo de gravidez & & & & & $<0,001$ \\
\hline Única & 314 & 1,0 & 1,00 & - & \\
\hline Múltipla & 18 & 2,2 & 2,30 & $(1,44-3,67)$ & \\
\hline Parto & & & & & 0,001 \\
\hline Vaginal & 125 & 0,8 & 1,00 & - & \\
\hline Cesáreo & 207 & 1,2 & 1,47 & $(1,18-1,83)$ & \\
\hline Sexo do recém-nascido & & & & & 0,003 \\
\hline Feminino & 136 & 0,8 & 1,00 & - & \\
\hline Masculino & 194 & 1,2 & 1,38 & $(1,11-1,72)$ & \\
\hline Prematuridade & & & & & $<0,001$ \\
\hline Não & 261 & 0,9 & 1,00 & - & \\
\hline Sim & 71 & 2,1 & 2,35 & $(1,81-3,05)$ & \\
\hline Baixo peso ao nascer & & & & & $<0,001$ \\
\hline Não & 256 & 0,8 & 1,00 & - & \\
\hline Sim & 76 & 2,5 & 2,88 & $(2,23-3,71)$ & \\
\hline Apgar insatisfatório ao $5^{\circ}$ min. & & & & & $<0,001$ \\
\hline Não & 294 & 0,9 & 1,00 & - & \\
\hline Sim & 38 & 4,2 & 4,63 & $(3,32-6,45)$ & \\
\hline
\end{tabular}

Fonte: SINASC/Ministério da Saúde.

Após a análise bivariada, foi construído um modelo de regressão logística multivariado, ajustado por todas as variáveis que apresentaram valor de $\mathrm{p}$ menor que 0,1 (valor de $\mathrm{p}<0,1$ ) na Tabela III. No modelo saturado, observou-se que a associação entre anomalias congênitas e as variáveis "tipo de gravidez" (valor de $p=0,488$ ) e "prematuridade" (valor de $p=0,500)$ não se mantiveram estatisticamente significantes. No modelo final, multivariado, a presença de anomalia congênita se manteve associada, de modo estatisticamente significante (valor de $p<0,01$ ), com a "idade materna", o "tipo de parto", o "sexo do recém-nascido", o "baixo peso ao nascer" e "escore de Apgar insatisfatório ao quinto minuto" de modo estatisticamente significante (valor de $\mathrm{p}<0,01$ ). Após a construção dos dois modelos, constatou-se, a partir do teste qui-quadrado de Pearson para ajuste da qualidade do modelo (valor de $\mathrm{p}=0,333$ ) e do teste da razão de verossimilhança (valor de $\mathrm{p}=0,598$ ), que o modelo final apresentou bom ajuste dos dados (Tabela IV). 
Tabela IV - Análise multivariada da associação entre anomalia congênita e idade materna, aspectos da gravidez e do parto e características dos recém-nascidos de Salvador, Bahia, 2014.

\begin{tabular}{|c|c|c|c|c|c|c|}
\hline & \multicolumn{2}{|c|}{ Modelo saturado } & \multicolumn{4}{|c|}{ Modelo final $*, * *$} \\
\hline & OR & IC & valor de $p$ & OR & IC & valor de $p$ \\
\hline Idade da mãe & & & 0,005 & & & 0,005 \\
\hline 18-35 anos & 1,00 & - & & 1,00 & - & \\
\hline$<18$ anos ou $>35$ anos & 1,69 & $(1,17-2,43)$ & & 1,69 & $(1,17-2,43)$ & \\
\hline Tipo de gravidez & & & 0,488 & & - & \\
\hline Única & 1,00 & - & & & & \\
\hline Múltipla & 1,20 & $(0,72-2,00)$ & & & & \\
\hline Parto & & & 0,001 & & & 0,001 \\
\hline Vaginal & 1,00 & - & & 1,00 & - & \\
\hline Cesáreo & 1,45 & $(1,16-1,82)$ & & 1,46 & $(1,17-1,83)$ & \\
\hline Sexo do recém-nascido & & & 0,003 & & & 0,003 \\
\hline Feminino & 1,00 & - & & 1,00 & - & \\
\hline Masculino & 1,40 & $(1,12-1,74)$ & & 1,40 & $(1,12-1,74)$ & \\
\hline Prematuridade & & & 0,500 & & & \\
\hline Não & 1,00 & - & & & & \\
\hline Sim & 1,14 & $(0,78-1,66)$ & & & & \\
\hline Baixo peso ao nascer & & & $<0,001$ & & & $<0,001$ \\
\hline Não & 1,00 & - & & 1,00 & - & \\
\hline $\operatorname{Sim}$ & 2,10 & $(1,45-3,03)$ & & 2,34 & $(1,77-3,08)$ & \\
\hline Apgar insatisfatório & & & $<0,001$ & & & $<0,001$ \\
\hline Não & 1,00 & - & & 1,00 & - & \\
\hline Sim & 3,27 & $(2,25-4,75)$ & & 3,34 & $(2,30-4,82)$ & \\
\hline
\end{tabular}

\section{DISCUSSÃO}

A prevalência de malformações congênitas nos nascidos vivos encontrada no presente estudo ficou abaixo do percentual estimado no mundo ${ }^{(3)}$, contudo, apresentou-se semelhante aos achados nas cidades de São Paulo ${ }^{(8)}$ e Pelotas ${ }^{(14)}$. A diferença entre a prevalência de anomalias congênitas do atual estudo com os achados internacionais relaciona-se a diversos fatores externos ao binômio mãe-bebê. Um desses fatores pode ser a fonte dos dados utilizada nos estudos, já que pesquisas feitas em hospitais de referência para gestantes de alto risco podem receber os casos com diagnóstico prévio de malformação, tendendo a apresentar maior proporção de bebês com anomalias ${ }^{(15)}$.

No contexto de estudos com dados secundários no país, destaca-se a melhoria na qualidade dos dados do SINASC, que se reflete num preenchimento mais completo e confiável da Declaração de Nascidos Vivos (DNV). Ainda que seja notável a melhoria na qualidade do preenchimento dos dados das DNV, entretanto, é importante destacar que a subnotificação de casos de malformações congênitas ainda ocorre no Brasil, levando, em alguns casos, à taxas inferiores à realidade ${ }^{(16)}$.

Ao se analisar as principais causas das malformações, percebeu-se que as maiores frequências foram de anomalias congênitas relacionadas ao sistema osteomioarticular e defeitos na formação do sistema nervoso. Os achados na literatura corroboram os encontrados do atual estudo, já que tais anomalias são mais facilmente detectadas ao exame físico logo após o nascimento $^{(17)}$.

Em relação às características maternas no presente estudo, a realização de parto cesárea e a presença de mães em extremos de idade foram variáveis que se associaram à anomalia congênita em recém-nascidos. Apesar da contradição na literatura quanto às indicações de parto cesárea para as anomalias congênitas, a associação entre as altas taxas de cesariana está relacionada à necessidade de assistência de maior complexidade ao recém-nascido, incluindo intervenções cirúrgicas no período neonatal ${ }^{(18)}$. Assim como observado neste estudo, em diferentes regiões do país encontrou-se associação positiva entre anomalia congênita e a realização de parto cesárea ${ }^{(19)}$, o que pode estar relacionado à indicação médica devido ao diagnóstico intrauterino de malformação.

No presente estudo, notou-se elevado percentual de cesáreas realizadas em Salvador. Tendo em vista a grande proporção de partos cesáreas no Brasil ${ }^{(20)}$ e as complicações associadas à sua realização, o Conselho Federal de Medicina (CFM) definiu, por meio da Resolução $n^{\circ} 2.144$ de 2016, que o parto cesárea só poderá ser realizado mediante os seguintes critérios: gestante informada quanto ao procedimento, seus benefícios e riscos; preenchimento do Termo de Consentimento Livre e Esclarecido; e 
idade gestacional maior ou igual a 39 semanas $^{(21)}$. Essa iniciativa poderá reduzir o percentual de partos cesariana realizados no país e os desfechos negativos que acometem mães e bebês associados a essa prática quando feita de modo inoportuno.

Diversos estudos evidenciam a idade avançada da gestante (acima de 35 anos) como fator de risco para a presença de anomalia congênita em recém-nascidos ${ }^{(22,23)}$. No presente estudo, observou-se maior prevalência de malformação em bebês filhos de mães em extremos de idade. Tais resultados são compatíveis com os achados de uma pesquisa realizada no Chile, entre os anos de 1996 a 2005, que observou aumento no risco de anomalias congênitas entre gestantes menores de 19 anos e maiores de 40 anos quando comparadas às gestantes de 25 a 29 anos $^{(24)}$. De modo geral, esse conjunto de evidências pode indicar relação entre o extremo da idade da gestante como proxy de aspectos biológicos que aumentam o risco de complicações na gravidez.

A presente pesquisa evidenciou que a ocorrência de anomalias congênitas foi maior em neonatos de baixo peso e escore de Apgar insatisfatório no quinto minuto, mesmo após o ajuste pelas variáveis ligadas à mãe, ao parto e pré-natal. Além disso, o atual estudo apontou maiores prevalências de anomalias em bebês do sexo masculino, resultado que coaduna com outros achados na literatura ${ }^{(8)}$. Ressalta-se ainda que a prematuridade não esteve associada à presença de anomalias em recém-nascidos no modelo multivariado. Apesar disso, a análise bivariada apontou maior prevalência de prematuridade em neonatos com alguma anomalia, resultado semelhante ao descrito por um estudo realizado em maternidades do Rio de Janeiro ${ }^{(25)}$.

A associação entre presença de malformações congênitas e desfechos adversos ao nascimento, como baixo peso ao nascer e escore de Apgar insatisfatório ao quinto minuto de vida, é bem relatada na literatura científica. Pesquisa realizada no México evidenciou que a média do peso ao nascimento foi menor em recém-nascidos com anomalia congênita e ainda que essa diferença era maior em alguns tipos de malformações, como aquelas relacionadas ao sistema digestivo e musculoesquelético ${ }^{(26)}$. Tanto os estudos transversais quanto os caso-controle têm demonstrado que o baixo peso ao nascer se associa a maior ocorrência de anomalias congênitas em países africanos ${ }^{(6,27)}$. No Brasil, estudos com distintas abordagem metodológicas apontam que neonatos com diagnóstico de anomalias congênitas apresentam mais chances de apresentar baixo peso ao nascer e escore de Apgar insatisfatório ao nascimento ${ }^{(28)}$.

O aumento na proporção de baixo peso e problemas respiratórios ao nascimento, indicado pelo escore insatisfatório de Apgar ao quinto minuto, podem ser explicados pelos efeitos da malformação no desenvolvimento intrauterino do neonato, já que há evidência que defeitos na formação do tubo neural estão associados com baixo peso e baixa vitalidade ${ }^{(29)}$. Outra hipótese que pode ser considerada para explicar essa associação é que bebês com desfechos adversos ao nascimento costumam ser tratados em unidades de terapia intensiva, o que aumentaria a probabilidade de uma investigação mais detalhada de suas condições de saúde e, consequentemente, a descoberta e registro da anomalia congênita ${ }^{(29)}$.

Aponta-se como limitação do estudo a utilização de dados secundários do Sistema de Informação sobre Nascidos Vivos, já que foge ao controle dos pesquisadores a qualidade do preenchimento das declarações de nascidos vivos que alimentam esse sistema. Sendo assim, optou-se pela exclusão das DNVs que não continham o preenchimento das variáveis utilizadas neste estudo, implicando em perda de informações. Apesar dessas perdas, o processo de limpeza manteve o mesmo total de observações, tanto na análise descritiva quanto na bivariada e multivariada. Outro ponto a ser destacado é que, por se tratar de um estudo transversal, a identificação de fatores associados não garante que tais variáveis possam ser consideradas fatores de risco. Em que pese tais limitações, a opção pelo uso dos microdados do SINASC, em substituição às metodologias mais tradicionais que investigam as anomalias a partir de estudos ecológicos ou com amostras menores, permitiu o acesso à quase totalidade de registros de nascimento, facilitando a operacionalização de um estudo transversal aplicado a um desfecho raro.

Os achados da presente investigação apontam a necessidade da oferta de cuidado especializado a esses recém-nascidos, prestado em unidades de terapia intensiva, de modo a evitar tanto a mortalidade quanto surgimento de outras morbidades no período neonatal.

A recente epidemia de Zika Vírus no Brasil ${ }^{(9,10)}$, seguida do aumento do número de nascimentos de bebês com microcefalia, são eventos que alertam para a necessidade de efetivação de políticas de atenção à saúde materno-infantil, com especial destaque à temática das malformações congênitas. Dentre essas políticas, destaca-se a Rede Cegonha, que precisa ser expandida a fim de garantir assistência de qualidade ao binômio mãe-bebê, incluindo cuidado adequado no pré-natal, parto e puerpério.

\section{CONCLUSÃO}

Os resultados indicam baixa prevalência de malformações congênitas, sendo esse desfecho associado às mães em extremo de idade e quadro clínico adverso ao nascimento, como baixo peso e Apgar insatisfatório.

\section{REFERÊNCIAS}

1. Mashuda F, Zhuechner A, Chalya PL, Kidenya BR, Manyama M. Pattern and factors associated with congenital anomalies among young infants admitted at Bugando Medical Centre, Mwanza, Tanzania. BMC Res Notes. 2014; 7:195.

2. World Health Organization. Congenital anomalies. Fact sheet n 370. 2015 [acesso em 2016 Set 08]. Disponível em: http:// www.who.int/mediacentre/factsheets/fs370/en/ 
3. Czeizel, AE. The primary prevention of birth defects: Multivitamins or folic acid? Int J Med Sci. 2004;1(1):50-61. .

4. Ministério da Saúde (BR), Secretaria de Atenção à Saúde. Diretrizes para Atenção Integral às Pessoas com Doenças Raras no Sistema Único de Saúde - SUS. Brasília: Ministério da Saúde; 2014 [acesso em 2016 Set 08]. Disponível em: http:// conitec.gov.br/images/Protocolos/Diretrizes_Atencao-DoencasRaras.pdf

5. Rocha RS, Bezerra SC, Lima JWO, Costa FS. Consumo de medicamentos, álcool e fumo na gestação e avaliação dos riscos teratogênicos. Rev Gaúch Enferm. 2013;34(2):37-45.

6. Kishimba RS, Mpembeni R, Mghamba J. Factors associated with major structural birth defects among newborns delivered at Muhimbili National Hospital and Municipal Hospitals in Dar Es Salaam, Tanzania 2011 - 2012. Pan Afr Med J. 2015; 20:153.

7. Feng Y, Yu D, Yang L, Da M, Wang Z, Lin Y, et al. Maternal lifestyle factors in pregnancy and congenital heart defects in offspring: review of the current evidence. Ital J Pediatr. 2014;40:85.

8. Mendes CQS, Avena MJ, Mandetta MA, Balieiro MMFG. Prevalência de nascidos vivos com anomalias congênita no município de São Paulo. Rev Soc Bras Enferm Ped. 2015;15(1):7-12.

9. Rodrigues LC. Microcephaly and Zika virus infection. Lancet. 2016;387(10033):2070-2.

10. Teixeira MG, Costa MCN, Oliveira WK, Nunes ML, Rodrigues LC. The Epidemic of Zika Virus-Related Microcephaly in Brazil: detection, control, etiology, and future scenarios. Am J Public Health. 2016;106(4):601-5.

11. Paixão ES, Barreto F, Teixeira MG, Costa MCN, Rodrigues LC. History, epidemiology, and clinical manifestations of zika: a systematic review. Am J Public Health. 2016;106(04):106-12.

12. Oliveira MM, Andrade SCAA, Dimech GS, Oliveira GCG, Malta DC, Rabelo DL Neto, Moura L. Avaliação do Sistema de Informações sobre Nascidos Vivos. Brasil, 2006 a 2010. Epidemiol Serv Saúde. 2015;24(4):629-40.

13. Luquetti DV, Koifman RJ. Qualidade da notificação de anomalias congênitas pelo Sistema de Informações sobre Nascidos Vivos (SINASC): estudo comparativo nos anos 2004 e 2007. Cad Saúde Pública. 2010;26(9):1756-65.

14. Castro MLS, Cunha CJ, Moreira PB, Fernandez RR, Garcias GL, Roth MGM. Frequência das malformações múltiplas em recém-nascidos na Cidade de Pelotas, Rio Grande do Sul, Brasil, e fatores sócio-demográficos associados. Cad Saúde Pública. 2006;22(5):1009-15.

15. Brito VRS, Sousa FS, Gadelha FH, Souto RQ, Rego ARF, França ISX. Malformações congênitas e fatores de risco materno em Campina Grande-Paraíba. Rev Rene. 2010;11(2):1-212.

16. Nicola PDR, Cernach MCSP, Perez ABA, Brunoni D. A utilização da Internet na notificação dos defeitos congênitos na Declaração de Nascido Vivo em quatro maternidades públicas do Município de São Paulo, Brasil. Cad Saúde Pública. 2010;26(7):1383-90.

17. Fontoura FC, Cardoso MVLML. Associação das malformações congênitas com variáveis neonatais e maternas em Unidades Neonatais numa cidade do Nordeste Brasileiro. Texto \& Contexto Enferm. 2014;23(4):907-14.

18. Peen GS. Indications for caesarean section. Best Pract Res Clin Obstet Gynaecol. 2001; 15(1):1-15.

19. Pinto CO, Nascimento LFC. Estudo de prevalência de defeitos congênitos no Vale do Paraíba Paulista. Rev Paul Pediatr. 2007;25(3):233-9.

20. Domingues RMSM, Dias MAB, Pereira MN, Torres JA, d Orsi E, Pereira APE, et al. Processo de decisão pelo tipo de parto no Brasil: da preferência inicial das mulheres à via de parto final. Cad Saúde Pública. 2014; 30(Supl 1):101-16.

21. Brasil. Conselho Federal de Medicina. Resolução nº 2144, de 17 de março de 2016. Diário oficial [da] República Federativa do Brasil. 2016 jun 22; Seção 1. p. 1677-7042.

22. Cleary-Goldman J, Malone FD, Vidaver J, Ball RH, Nyberg DA, Comstock CH, et al.. Impact of maternal age on obstetric outcome. Obstet Gynecol. 2005;105(5 Pt 1):983-90.

23. Guerra FAR, Llerena JCJ, Gama SGN, Cunha CB, Theme MMF. Defeitos congênitos no Município do Rio de Janeiro, Brasil: uma avaliação através do SINASC (2000-2004). Cad Saúde Pública. 2008;24(1):140-9.

24. Nazer J, Cifuentes O, Águila A, Ureta P, Bello MP, Correa F, et al. Edad materna y malformaciones congénitas. Un registro de 35 años. 1970-2005. Rev Med Chile. 2007;135(11):1463-9.

25. Costa CMS, Gama CGN, Leal MC. Malformações congênitas no Município do Rio de Janeiro, Brasil: prevalência e fatores associados a sua ocorrência. Cad Saúde Pública. 2006;22(11):2423-31. 
26. Montes-Núñez S, Chávez-Corral DV, Reza-López S, Sanin LH, Acosta-Maldonado B, Levario-Carrilho M. Birth Weithg in Children With Birth Defects. Birth Defects Res A Clin Mol Teratol. 2011; 91(2):102-7.

27. Shawky RM, Sadik DI. Congenital malformations prevalent among Egyptian children and associated risk factors. Egyptian J Medical Human Genetics. 2011; 12:69-78.

28. Nhoncanse GC, Germano CMR, Avó LRS, Melo DG. Aspectos maternos e perinatais dos defeitos congênitos: um estudo caso-controle. Rev Paul Pediatr. 2014;32(1):24-31.

29. Nascimento LFC. Prevalência de defeitos de fechamento de tubo neural no Vale do Paraíba, São Paulo. Rev Paul Pediatr. 2008;26(4):372-7.

\section{Endereço para correspondência:}

Elzo Pereira Pinto Junior

Instituto de Saúde Coletiva da Universidade Federal da Bahia

Rua Oito de Dezembro, 808/103

Bairro: Graça

CEP: 40150-000 - Salvador - BA - Brasil

E-mail: elzojr@hotmail.com 